\title{
Clinicopathological correlation of blistering diseases of skin
}

\author{
A.K.M. Nurul Kabir ${ }^{1}$, Mohammed Kamal ${ }^{1}$ and Aga Masood Choudhury ${ }^{2}$ \\ Department of ${ }^{1}$ Pathology, ${ }^{2}$ Dermatology and Venereology, Bangabandhu Sheikh Mujib Medical \\ University, Shahbag, Dhaka 1000, Bangladesh.
}

\begin{abstract}
Blistering diseases are alarming skin conditions where blister formation occurs in various ways and cannot be differentiated clinically. For confirmation of diagnosis, along with routine histological examination, immunofluorescence study is essential. Tzanck smear may be used as a rapid diagnostic tool. We included here 34 cases of different blistering lesions. Other than routine Hematoxylin \& Eosin stain, direct immunofluorescence test was done in 31 cases, indirect immunofluorescence in 28 cases and Tzanck smear in 33 cases. Direct immunofluorescence stain was also applied on Tzanck smears. The most frequent diagnoses were pemphigus $(n=16)$, bullous pemphigoid $(n=11)$ and linear IgA dermatosis $(n=3)$. Clinical findings and histological examination were sufficient for the diagnosis of most cases. Direct immunofluorescence study is essential in many cases, and indirect immunofluorescence study is a useful method for diagnosis of some of the blistering diseases, especially in pemphigus. Direct immunofluorescence staining on Tzanck smear is a novel technique for the diagnosis of pemphigus.
\end{abstract}

\section{Introduction}

Blistering (bullous) diseases are a heterogeneous group of dermatological disorders. These are worldwide common disorders and also occur in our country. In most cases the blisters (vesicles and bullae) are due to a primary disease e.g. pemphigus. As a secondary phenomenon it also occurs in a number of unrelated conditions. Five principal ways can result in blister formation namely: genetic derangement, physical, immunological and inflammatory damage and drug reaction ${ }^{1}$. Of these, immunological reaction accounts for most of the dermatological diseases producing blisters.

In many cases, bullous diseases cannot be differentiated clinically and needs the help of histopathological examination and immunofluorescence study. Sometimes examination of blister fluid can be used. Routine histological examination can help in the diagnosis of most of the blistering cases according to location and morphology of blisters. Most immune mediated blistering diseases can be defined on the basis of both direct and indirect immunofluorescence findings, and the pattern obtained is often diagnostic.

Tzanck smear (a smear made by scrapped material from the floor and roof of an early, freshly opened blister) is an acceptable tool for diagnosis of blistering diseases ${ }^{2}$. The method has certain advantages as a rapid aid to the diagnosis of blistering diseases.

\section{Materials and Methods}

This study was done during the period of March 2001 to June 2002 with an aim to determine the clinicopathlogical correlation of blistering diseases by routine and direct immunofluorescence staining of lesional and perilesional skin. Study with indirect immunofluorescence technique and characters of cells obtained by Tzanck smear are also studied. The patients were selected from the in and outpatient department of Dermatology of BSMMU and Bangladesh Institute of Research on Diabetes, Endocrinology and Metabolism (BIRDEM) Hospital, Dhaka.

Patients were selected with the following inclusion criteria: any patient with intact blisters, all age and sex groups, giving verbal consent for the study. Exclusion criterion was patient who refused to be included in the study protocol.

Clinical information were obtained by a brief history taking, general and dermatological examination. A total 34 cases with history of blistering lesion were included. Other than routine 
staining direct immunofluorescence was done in 31 cases, indirect immunofluorescence study in 28 and Tzanck smear preparations studied in 33 cases. Skin biopsy sample was obtained containing a small intact bulla for routine sectioning and was placed in $10 \%$ formalin and perilesional skin for direct immunofluorescence staining placed in normal saline. Formalin fixed sample processed routinely and stained with Hematoxylin and Eosin stain. Tissue samples (perilesional skin) collected in normal saline were quickly frozen and 4-5 $\mu \mathrm{m}$ thick sections were cut in the cryostat (Minotome, International Equipment Company, Damon, USA) at $-20^{\circ} \mathrm{C}$ and were air-dried and kept at $-20^{\circ} \mathrm{C}$ until staining. The sections were washed in phosphate buffered saline and incubated with conjugates (FITC conjugated rabbit anti-human IgG, IgA, IgM, C3 and fibrinogen; MEDIC, Italy).

Blood for serum and blister fluid were taken from same patient and stored at $-20^{\circ} \mathrm{C}$. Normal human skin was obtained from patients undergoing surgical operation and urinary bladder of Long Evans rat were collected and used as substrate. Cryostat sections of normal human skin were incubated with serum or blister fluid (diluted in phosphate buffered saline 1:5 and 1:10) of patients for $30 \mathrm{~min}$. Normal serum was included as control. Rest of the procedure was as for direct immunofluorescence. Serum of one case (paraneoplastic pemphigus) was studied using both normal human skin and rat urinary bladder as substrates.

Tzanck smears were prepared from blister after selecting an early typical vesicle or bulla. The base and under surface of the roof were gently scraped with the sharp edge of blade. Smears were made for Papanicolaou and Giemsa stain and for direct immunofluorescence examination. The smears were stained routinely by both Papanicolaou's and Giemsa methods and were examined under light microscope for identification of acantholytic cells and inflammatory cells. For direct immunofluorescence staining air-dried slides were stored at $-20^{\circ} \mathrm{C}$ and later stained with conjugates (anti-IgG, IgA and IgM) using same procedure as for skin tissue.

All the slides for direct immunofluorescence and indirect immunofluorescence study were examined under an incident (epi-illumination) fluorescence microscope (Hertel and Reuss, Germany with Exciter filter BP485 and Barrier BP520).

\section{Results}

Among the 34 cases, 19 (55.88\%) were male and 15 (44.11\%) were female. Male to female ratio was
1.26:1. The age ranged from 5-75 years with mean age 35.1 years ( $\mathrm{SD} \pm 19.8$ ). The maximum numbers of patients $(n=14,41.16 \%)$ were in fourth and fifth decades. In this study the largest number $(n=16)$ of patients were in pemphigus group. The other important diagnoses were bullous pemphigoid $(n=11)$ and linear IgA dermatosis $(n=3)$. The pemphigus group included pemphigus vulgaris $(n=8)$, pemphigus foliaceus $(n=7)$ and one case of paraneoplastic pemphigus. Histological findings of seven cases of pemphigus vulgaris were suprabasal blisters with acantholytic cells and variable number of neutrophils and eosinophils and in one case no blister was seen in routine sections. Pemphigus foliaceus cases showed subcorneal blisters with acantholytic cells. The paraneoplastic pemphigus case showed both suprabasal and subepidermal blisters. Out of eight cases of pemphigus vulgaris, seven (87.5\%) showed epidermal intercellular deposition of IgG and one (12.5\%) showed granular deposition of C3 along the basement membrane zone. Among seven cases of pemphigus foliaceus, direct immunofluorescence study was done in six cases. Intercellular deposition of IgG was seen in all cases (100\%) along with intercellular depositions of C3 in two (33.33\%) cases and focal basement membrane zone deposition of C3 and IgM in one (16.66\%) case. The paraneoplastic case with clinical history of non-Hodgkin's lymphoma, revealed depositions of IgA and C3 along the basement membrane zone with depositions of IgG both in epidermis and basement membrane zone in direct immunofluorescence test.

The next common diagnosis was bullous pemphigoid with findings of subepidermal blisters containing large number of eosinophils and few neutrophils. Out of 11 cases of bullous pemphigoid, 10 cases studied by direct immunofluorescence and five (50\%) cases showed depositions of C3, four (40\%) cases showed C3 and IgG, and one (10\%) case showed C3, IgG and IgM along the basement membrane zone. Three cases, with subepidermal blisters in histological sections, showed linear depositions of IgA along the basement membrane zone in direct immunofluorescence and diagnosed as linear IgA dermatosis. One case of erythema multiforme with intraepidermal blister containing necrotic cells showed granular deposition of C3 in dermal blood vessel walls. Clinically diagnosed a case as epidermolysis bullosa dystrophica proved by direct immunofluorescence as epidermolysis bullosa acquisita, showing granular deposition of C3 along the basement membrane zone. One case, clinically suspected as chronic bullous disease of childhood, confirmed histologically as subacute dermatitis. One case with clinical and histological 
diagnosis of epidermolysis bullosa showed no immune deposition in direct immunofluorescence stain.

In indirect immunofluorescence study, out of five cases of pemphigus vulgaris, four (80\%) showed epidermal intercellular deposition of IgG. Among seven cases of pemphigus foliaceus all (100\%) showed epidermal intercellular deposition of IgG. Indirect immunofluorescence test was positive in paraneoplastic pemphigus, as with direct immunofluorescence, showing linear deposition of IgA along basement membrane zone of both human skin and rat urinary bladder. In bullous pemphigoid, 40\% cases showed IgG and in linear IgA dermatosis 33.33\% case IgA deposition along the basement membrane zone. These findings were compared with direct immunofluorescence result and showed good concordance (Table I).

Table I: Indirect immunofluorescence (IIF) findings in pemphigus group- compared with Direct immunofluorescence (DIF) results



In Tzanck smear study pemphigus vulgaris and pemphigus foliaceus cases showed acantholytic cells mixed with variable number of inflammatory cells in routine staining (Figure 1). The acantholytic cells are epithelial cells lying in groups or singly. Many of the cells seem to be detached or loosely attached to neighboring cells rather than in a tightly adherent sheet. The typical acantholytic cell (Tzanck cell or Tzanck-like cell) contains a large, centrally located, hyperchromatic nucleus with prominent nucleolus. Moderate to large number of cells were present in pemphigus vulgaris and pemphigus foliaceus. Two cases of bullous pemphigoid and the subacute dermatitis cases showed regenerated epithelial cells. In bullous pemphigoid important cells were eosinophils and variable mixture of neutrophils, lymphocytes and macrophages. In direct immunofluorescence study of smears, out of eight cases of pemphigus vulgaris, deposition of IgG on and around individual cells was positive in 7 (87.5\%) cases and in pemphigus foliaceus it was positive in all of the 6 (100\%) cases studied (Figure 2). No deposition was seen in remaining 20 cases including one case of pemphigus vulgaris (Table II).



Figure 1: Photomicrograph of Tzanck smear from a case of Pemphigus vulgaris. The acantholytic cells are loosely attached. Scattered lymphocytes and eosinophils are also seen (Papanicolaou stain, x 800)

\section{Discussion}

The keratinocytes of the epidermis are tightly bound together by desmosomes and intercellular substances. Beneath the epidermis lies the basement membrane zone, a special area of cellextracellular matrix adhesion. Antibodies may target against many of the component in these loca 


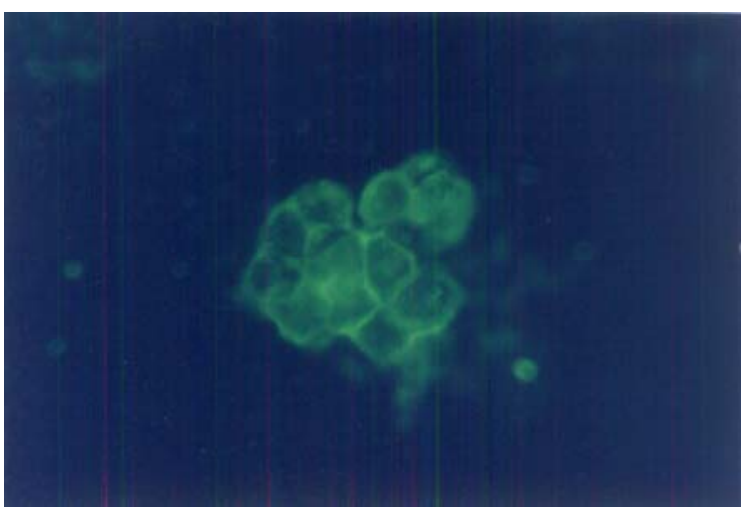

Figure 2: Photomicrograph of direct immunofluorescence stain on Tzanck smear from a case of pemphigus foliaceus showing deposition of IgG on the cell surface and in the intercellular substance of acantholytic cells (Rabbit anti-human IgG-FITC conjugate, $\mathrm{x}$ 1600). tions e.g. desmoglein-III and desmoglein-I antigens of pemphigus vulgaris and pemphigus foliaceus respectively or bullous pemphigoid antigen. Deposition of antibody can be demonstrated at the level of blister formation or at the site adjacent to blisters $^{1}$. The study was done to characterize different histological findings and to correlate with clinical diagnosis of blistering lesion.

In the present study, the maximum numbers of subjects $(41.17 \%)$ were in fourth and fifth decades and the disease occurs in adult and both sexes are affected equally. Out of 34 cases the largest group was pemphigus- consisting 16 (47.05\%). The second largest group was bullous pemphigoid $(\mathrm{n}=11,32 \%)$. Collier and Wojnarowska also presented a data with slight higher incidence of

Table II. Findings of Direct immunofluorescence (DIF) study on Tzanck smear

\begin{tabular}{|c|c|c|c|c|c|}
\hline Diagnosis & $\begin{array}{l}\text { No. of cases showing } \\
\text { deposits of IgG around } \\
\text { cell surface }\end{array}$ & $\begin{array}{l}\text { No. of cases showing no } \\
\text { deposits }\end{array}$ & $\begin{array}{l}\text { No. of cases } \\
\text { DIF done }\end{array}$ & $\begin{array}{l}\text { No. of cases } \\
\text { DIF not done }\end{array}$ & Total \\
\hline Pemphigus vulgaris & 7 (87.5\%) & $1(12.5 \%)$ & 8 & - & 8 \\
\hline Pemphigus foliaceus & $6(100 \%)$ & - & 6 & 1 & 7 \\
\hline Paraneoplastic pemphigus & - & 1 & 1 & - & 1 \\
\hline Bullous pemphigoid & - & 11 & 11 & - & 11 \\
\hline Linear IgA & - & 3 & 3 & - & 3 \\
\hline Erythema multiforme & - & 1 & 1 & - & 1 \\
\hline Epidermolysis bullosa acquisita & - & 1 & 1 & - & 1 \\
\hline Epidermolysis bullosa & - & 1 & 1 & - & 1 \\
\hline Subacute dermatitis & - & 1 & 1 & - & 1 \\
\hline Total & 13 & 20 & 33 & 1 & 34 \\
\hline
\end{tabular}

pemphigus followed by bullous pemphigoid ${ }^{1}$. Among the eight cases of pemphigus vulgaris, seven cases showed suprabasal cleft with acantholytic cells and variable number of inflammatory cells. Most of the cases reveled intercellular lace like deposition IgG. Pemphigus foliaceus cases showed subcorneal blister with acantholytic cells. All cases showed intercellular IgG and few cases with other deposits. Similar results were observed by Judd and Lever ${ }^{3}$. The case with history of non-Hodgkin's lymphoma showed both suprabasal and subepidermal blister and depositions were IgA and C3 along basement membrane zone. Cohen et al. mentioned a case of mixed bullous disease with B-cell lymphoma diagnosed as paraneoplastic pemphigus, where the depositions were intercellular IgM and IgG along basement membrane zone ${ }^{4}$. In bullous pemphigoid the sub-epidermal bullae contain small to moderate number of eosinophils and neutrophils and common deposit in direct immunofluorescence was C3. Provost et al. showed that deposition of C3 was present in all $(100 \%)$ of 11 cases, they studied ${ }^{5}$. The three cases of linear IgA dermatosis histologically revealed subepidermal bullae containing neutrophils and eosinophils. All of these revealed linear deposition of IgA along the basement membrane zone in direct immunofluorescence study. One of these cases also revealed focal deposition of fibrin along basement membrane zone. Cohen et al. mentioned that some patients with linear IgA deposits at the basement membrane zone are better classified as IgA bullous pemphigiod or IgA epidermolysis bullosa acquisita, as the antibodies have specificity for BPAg1 or type VII collagen respectively ${ }^{4}$. Mehregan also described that it is not yet clear whether these patients constitute a subgroup of 
atypical dermatitis herpetiformis or a variant of bullous pemphigoid ${ }^{6}$.

By indirect immunofluorescence study out of five cases of pemphigus vulgaris, 4 (80\%) showed epidermal intercellular deposition of IgG. All seven cases of pemphigus foliaceus (100\%) showed epidermal intercellular deposition of IgG. In paraneoplastic pemphiguses linear deposition of IgA was present along the basement membrane zone of both skin and rat urinary bladder indicating a distinct feature. In bullous pemphigoid, 40\% cases showed IgG and in linear IgA dermatosis 33.33\% case showed IgA deposition along the basement membrane zone. A study showed positive result of indirect immunofluorescence, 79- 90\% in pemphigus and $70 \%$ in bullous pemphigoid ${ }^{7}$.

For Tzanck smears both Papanicolaou's and Giemsa stains were used routinely in this study. The findings in both stains were more or less equal. In the present study the characteristic features were the presence of many acantholytic cells in the pemphigus vulgaris and pemphigus foliaceus. Smears from nine of 11 patients with bullous pemphigoid showed small to large number of eosinophils, neutrophils and occasionally lymphocytes. No acantholytic cells were seen in these smears. In two cases squamous epithelial cells were seen along with inflammatory cells. These represented regenerated epithelial cells at the blister base of older lesions. Epithelial cells or acantholytic cells were not seen in linear IgA dermatosis, epidermolysis bullosa acquisita and epidermolysis bullosa. These revealed only inflammatory cells such as neutrophils, eosinophils and lymphocytes. Blank and Burgoon also noted the most striking difference of pemphigus vulgaris from all other vesicular or bullous disease by demonstrating a large numbers of epithelial cells at base of early pemphigus bullae in contrast with the few epithelial cells obtained by scraping the base of other bullous diseases ${ }^{8}$. In addition to routine cytological examination, Tzanck smears were studied by direct immunofluorescence stain to detect antibodies present on the cell surfaces as acantholytic cells of pemphigus also bear antigens. The deposition occurs on the cell surface without staining the nucleus. In a study on five cases of pemphigus vulgaris Acosta and Ivanyi found intercellular IgG deposition on both biopsy specimen and cytological smears ${ }^{9}$. Among 16 pemphigus cases direct immunofluorescence test was done on smears of 15 patients of which 13 (86.66\%) cases showed deposition of IgG on the cell surface. The deposits were present on the individual cells or in the intercellular substances where the cells were present in clusters. Smears from 18 cases of other blistering diseases including 11 cases of bullous pemphigoid showed no immunodeposition. It is evident that a significant immunodeposition observed in pemphigus group, 88.66\% (Z test, $\mathrm{p}<.001$, highly significant). From these findings it implies that direct immunofluorescence study on Tzanck smear is a reliable test for pemphigus cases.

Diagnosis of blistering diseases can often made on the basis of clinical features but in some cases it may be possible to produce only differential diagnosis ${ }^{6}$. Less than $50 \%$ of clinical diagnoses show concordance with final diagnosis in present study. Direct immunofluorescence study has definitive role in distinguishing immune mediated blistering diseases from others. Some immunopathological patterns are disease specific, such as fishnet pattern of epidermal intercellular deposits are specific for pemphigus. The lesions with basement membrane zone depositions may be sometimes inconclusive and deposits along the epidermal-dermal junction can be found in many diseases, such as lupus erythematosus, epidermolysis bullosa acquisita and porphyria ${ }^{6}$. Direct and indirect immunofluorescence tests with split-skin studies enable the typing and localization of immunoglobulin deposits, which can be used in special situations ${ }^{10}$. Direct immunofluorescence technique, combined with routine histology, is a useful method in distinguishing most of the bullous diseases, if not all. Indirect immunofluorescence test is helpful commonly in pemphigus cases, frequently in bullouss pemphigoid and occasionally in linear IgA dermatosis. Indirect immunofluorescence test can be utilized as an alternative or preliminary test in these cases when direct immunofluorescence test could not be done as some patient may refuse biopsy and can be utilized also for the patients staying in remote area from where sera can be easily collected and transported. By Tzanck smears accurate diagnosis can be made in pemphigus group of diseases where acantholytic cells and/or typical Tzanck like cells are present. No acantholytic cells with predominant eosinophils indicate a case of bullous pemphigoid. But in most of cases with subepidermal blisters the smear findings are nonspecific. Before a definitive diagnosis to be made it requires clinical correlation with the disease. This is particularly helpful in differentiating the other various bullous diseases from pemphigus. The cytological differences among smears of pemphigus vulgaris and familial benign pemphigus are subtle. Even bullous diseases with sub epidermal blisters may contain small number of acantholytic cells. In these cases, pemphigus group of diseases can be definitely distinguished from other blistering diseases by 
direct immunofluorescence staining. It is a novel approach and can be of help in the diagnosis of blistering diseases.

It can be concluded that direct immunofluorescence study of perilesional skin is essential in the diagnosis of blistering diseases, indirect immunofluorescence study is a useful method for the diagnosis of some of the vesiculobullous diseases and Tzanck smear, combined with immunofluorescence stain, is a novel technique for the diagnosis of pemphigus.

\section{Acknowledgement}

We are grateful to Department of Immunology, BIRDEM, Dhaka for kindly providing two Long Evans rats free of cost.

\section{References}

1. Collier PM, Wojnarowska F. Blistering diseases. Med Int. 1997; 11: 51-53.

2. Graham JH, Bingul O, Burgoon CRJr. Cytodiagnosis of Inflammatory dermatoses. Arch Dermatol. 1963; 87: 118-27.

3. Judd KP, Lever WF. Correlation of antibodies in skin and serum with disease severity in pemphigus. Arch Dermatol 1979; 115: 428-32.
4. Cohen LM, Skopicki DK, Harrist TJ, Clark WH Jr. In: Lever's Histopathology of the Skin, Elder D, Elenitsas R, Jaworsky C, Johnson B (eds). $8^{\text {th }}$ ed. Philadelphia, Lippincott-Raven, 1997, pp 209-52.

5. Provost TT, Maize JC, Ahmed AR, Strauss JS, Dobson RL. Unusual subepidermal bullous diseases with immunological features of bullous pemphigoid. Arch Dermatol. 1997; 115: 156-60.

6. Mehregan AH. Pinkus' Guide to dermatohistopathology, $4^{\text {th }}$ ed. Connecticut, Appleton-Century-Crofts, 1986, pp 99-110.

7. Harrist TJ, Mihm MC. Cutaneous immunopathology: The diagnostic use of direct and indirect immunofluorescence techniques in dermatologic disease. Human Pathol. 1979; 10: 625-53.

8. Blank H, Burgoon, CF. Abnormal cytology of epithelial cells in Pemphigus vulgaris: A diagnostic aid. J Invest Dermatol. 1952; 18: 213-23.

9. Acosta E, Ivanyi L. Comparison of the reactivity of various epithelial substrates for the titration of pemphigus antibodies by indirect immunofluorescence. Br J Dermatol. 1982; 107: 537-41.

10. Wuepper KD. Repeat direct immunofluorescence to discriminate pemphigoid from epidermolysis bullosa acquisita. Arch Dermatol. 1990; 126: 1365. 